ent theories about what organizational phenomena are important; especially when one of the strategies is based on the same theory as, and indeed the results of, the questionnaire itself. It is absolutely valid, and very interesting, to use the questionnaire as one criterion, but at the very minimum it should be matched by one criterion measure derived from another theory of change.

By way of summary, I would note that almost all of my questions could have been answered briefly and unobtrusively in the article itself. Without these answers, some people will be falsely impressed by the vast numbers of the sample sizes, while others will dismiss the whole study as just another example of establishment-sponsored lying with statistics.

\author{
-William R. Torbert \\ Graduate School of Education \\ Harvard University
}

\title{
Back to Bowers on OD Techniques
}

Professor Torbert raises a number of challenging questions. Some reflect the fact that journal space limitations simply do not permit the presentation of the array of back-up material that might be useful to various readers. Others are large substantive issues which can at best be given brief treatment here. I shall attempt to respond to the points as numbered.

1. Our assumptions are obviously different. I do not agree with the view, which admittedly has some currency, that an organization is a relatively homogeneous entity which can be characterized by a grand mean. It is, instead, a social system whose functionally related "parts" are made up of nothing more than the variously aggregated, configured, and perceived behaviors of its members. Had the article been entitled, "OD Techniques and Their Impact upon 23 Social Systems," an entirely different analytic strategy would have been required, one which might or might not have involved presenting grand means. However, that was not the purpose of the article. Instead of focusing upon what changed behavior does to system outcomes, it treated the effects of OD techniques on behaviors within 23 organizations. The alternative question is, of course, a worthy one, but can scarcely be handled within the confines of a journal article.

In any attempt to satisfy, albeit meagerly, the curiosity of a number of readers concerning a "by organization" tally, a scanning of organizational totals results in the following general description:

a. Four of the five organizations which received the "Survey Feedback" treatment show overall improvement; one shows overall decline.

b. All five of the organizations which received the Laboratory Training treatment show overall declines.

c. Three of the four organizations which received the Interpersonal Process Consultation treatment show overall improvement; one declines.

d. Four of the five organizations which received the Task Process Consultation treatment show marginal overall declines; one shows substantial improvement.

e. One of the two organizations which received the Data Handback treatment shows overall improvement; one shows a decline. 
f. Both of the organizations which received No Treatment show an overall decline.

28:3. I will attempt to answer these points together. At the time when these analyses were conducted, approximately half of the organizations (10) had experienced a third major wave of survey data collection. In all instances, however, this third wave followed what is here termed the "postmeasure," and in no instance did it occur prior to the "premeasure." The basic strategy of our survey data collections involves a complete census (full coverage) of organizational members participating in the study. Where sampling was used, it consisted of a 20 per cent random sample from a population consisting of all participating members. A complete census is, of course, unattainable, and at any given point some percentage is missed because of illness, vacations, and the like. Our response percentage does not ordinarily miss the 100 per cent target by anything in excess of the 15 per cent figure which is mentioned by Professor Torbert.

4. One wishes, of course, that OD research efforts of the kind reported here could always take the form of nicely controlled field experiments. All too often they do not, and such was the case in this instance. They can, perhaps, best be thought of as "natural" experiments, in which the nature of the intervention "package" was determined largely by personal style preference of the change agent, with schedules (and events related to those schedules) largely determining which change agents assumed responsibility for which site. All of the principal change agents were staff members of the Institute for Social Research. They were in most instances assisted by persons selected from within the company participating in the project to form an "internal change agent" team.

A more critical question, I believe, is that raised as $4 \mathrm{c}$. One might imagine that handing back tabulated data with little, or only cursory, discussion could well lead to disillusionment and negative change for the mass of the organization's membership. Yet, as an explanation for the findings concerning, for example, laboratory training, this seems implausible for at least two reasons: (a) This bears some resemblance to what is here labeled "Data Handback," in which the effects were not all that negative. (b) It seems inadequate to explain the negative changes in the laboratory-trained Capstone groups themselves.

On at least one point I believe we can agree: more, and better, research on these issues is surely needed.

-David G. Bowers

Program Director, ISR

University of Michigan

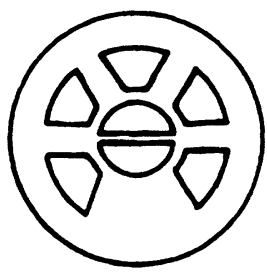

BACKFILE ISSUES of this journal are available on 35MM MICROFILM

University Microfilms

A Xerox Company Ann Arbor, Michigan 48106 Write for catalog and complete information. 